\title{
Analysis of Ultrasound Clinical Early Warning System in Hyper-Coiling Umbilical Cord
}

\author{
Junshu XIE \\ Peking University People's Hospital \\ Yani YAN \\ Peking University People's Hospital \\ Zhenjuan YANG \\ Peking University People's Hospital \\ Yuntao LI \\ Peking University People's Hospital \\ Xiaowei XUE \\ Peking University People's Hospital \\ Xiaohong ZHANG \\ Peking University People's Hospital \\ Qiuyan PEI ( $\sim$ pqypei@126.corn ) \\ Peking University People's Hospital
}

\section{Research Article}

Keywords: clinical early warning system, FGR, hyper-coiling umbilical cord, UCl, umbilical artery blood flow spectrum

Posted Date: October 18th, 2021

DOI: https://doi.org/10.21203/rs.3.rs-953223/v1

License: (c) (1) This work is licensed under a Creative Commons Attribution 4.0 International License. Read Full License 


\section{Abstract}

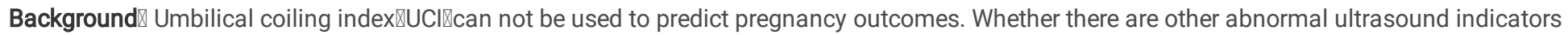
in the hyper-coiling cord $(\mathrm{HC})$ that can give early warning of adverse pregnancy still needs to be further explored.

Objective: To establish an ultrasonic early warning system for clinical intervention in patients with the hyper-coiling umbilical cord.

Study Design: Retrospectively analyzed 29 patients' characteristics with hyper-coiling cord (HC) diagnosed by ultrasound in our hospital from January 2019 to March 2021. According to whether the following high-risk factors were combined 邓High resistance of umbilical arteries beside the

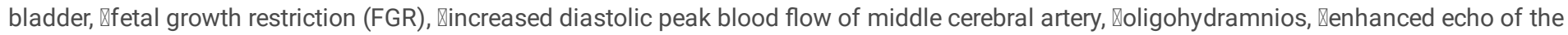
cerebral parenchyma, Zreversed A wave of the venous catheter (VC). It could be divided into two types: simple type and complex type, and then divided into three groups according to the delivery situation of patients, as terminated pregnancy group, intervention group, and non-intervention group. At last, according to the distribution of high-risk factors in each group, established an ultrasonic early warning system which affected the clinical intervention and pregnancy outcome of complex HC patients.

Results: compared with 9 cases in the simple $\mathrm{HC}$ group and 20 cases in the complex $\mathrm{HC}$ group, the intervention ratio of complex $\mathrm{HC}$ patients was higher $(p=0.027)$, the average delivery gestational weeks was smaller $(p=0.034)$, the abnormal rate of fetal heart monitoring was higher $(p=0.027)$, and there were statistical differences in above. Compared in the three groups (terminated pregnancy group, intervention group, and the nonintervention group), there were statistical differences in the number of high-risk factors $(\mathrm{P}=0.000)$ and the distribution proportion of six high-risk factors $(P=0.012,0.011,0.000,0.026,0.028,0.000)$.

Conclusion: the monitoring of six high-risk factors by ultrasound could establish an ultrasound early warning system for HC to influence the clinical intervention and pregnancy outcome. In this early warning system, the monitoring of fetal intrauterine safety was often needed for complex HC patients. High resistance of umbilical arteries beside the bladder and FGR were the earliest warning signs. Once timely clinical intervention was carried out, the prognosis of pregnancy could be improved.

\section{Introduction}

The umbilical cord is the vital link between the fetus and placenta which carries oxygenated blood to the fetus via the umbilical vein and removes deoxygenated blood via the umbilical arteries. The proper spiral state of the umbilical cord plays a key role in maintaining its function. The umbilical coiling index $(\mathrm{UCl})$, defined by Strong et al, is the number of the spiral cycles every $1 \mathrm{~cm}$ length, which is normally 0.17 , and $\mathrm{UCl}<0.07$ is considered as hypo-coiling, more than 0.30 is considered as hyper-coiling ${ }^{[1]}$.

Some literature thinks that hyper-coiling umbilical cord was found to be significantly associated with intrauterine growth restriction, low birth weight, oligohydramnios, abnormal fetal heart rate monitoring, preterm labor pains, and instrumental vaginal delivery [2]. However, many other studies have failed to find a strong correlation between abnormal coiling and adverse outcomes ${ }^{[3]}$. In their review, Sebire concluded that the majority of pregnancies with abnormal coiling have normal outcomes ${ }^{[4]}$. Therefore, whether there are other abnormal ultrasound indicators in the hyper-coiling cord $(\mathrm{HC})$ that can give early warning of adverse pregnancy, to intervene clinical treatment to improve the pregnancy outcome, still needs to be further explored.

This study aimed to review our experience with the prenatal diagnosis of the hyper-coiling umbilical cord, and find out which ultrasound indicators are abnormal indicates needed for clinical intervention or is related to the adverse outcome of pregnancy. To our knowledge, this is the first study to establish an early-warning system of ultrasound that affects the clinical intervention and pregnancy outcome in the hyper-coiling umbilical cord.

Also,this was the first time that we used the diastolic notch of bilateral umbilical arteries of the bladder to judge the resistance state of the umbilical cord. On the one hand, compared with the resistance index (RI), pulsatility index (PI) and maximum systolic blood flow velocity and diastolic blood flow velocity in the lowest value ratio(S/D) of the umbilical cord, the diastolic notch of umbilical arteries can more objectively reflect the resistance state of the umbilical cord, which was not affected by artificial factors and measurement factors. On the other hand, compared with the isolated segment of the umbilical cord, the umbilical arteries on both sides of the bladder were located at the beginning of the umbilical cord, which can better reflect the overall resistance state of the whole umbilical cord without the influence of the spiral position.

\section{Materials And Methods}

This study is a single center retrospective study.

1. Patients 
We summarized 38 cases of hyper-coiling umbilical cord diagnosed in our hospital from January 2019 to March 2021 whose UCl was more than 0.60 , except for severe obstetric complications such as preeclampsia, gestational diabetes mellitus (GDM), twin pregnancy, pregnancy with immune system diseases, viral infection, fetal chromosomal abnormalities or structural malformations and fetal distress caused by other reasons. Finally, 29 patients with hyper-coiling umbilical cord without other complications were retained. And all patients conformed hyper-coiling umbilical cord after birth by measured UCl.

\section{Ultrasound examination}

The routine fetal ultrasound scan was performed following the practice guidelines of the International Society of Ultrasound in Obstetrics and Gynecology ${ }^{[5]}$, using a Voluson E8 Expert machine (GE Healthcare, Kretzchnik). The growth and development parameters (including biparietal diameter, head circumference, abdominal circumference, and femur length. i.c. BPD, HC, AC, FL), amniotic fluid volume, placental position, umbilical cord, and its attachment point were recorded.

\section{Classification of hyper-coiling umbilical cord}

We would measure the $\mathrm{UCl}$ of suspected hyper-coiling umbilical cord patients and record. When $\mathrm{UCl}>0.60$, it can be diagnosed as the hypercoiling umbilical cord. Then the following examinations were performed according to the routine of our hospital. Whether combined or not one of the following 6 high-risk factors, patients were divided into the complex and simple hyper-coiling umbilical cord. After that regular ultrasound reexamination was conducted in our hospital every $2 \sim 4$ weeks. Once diagnosed as hyper-coiling umbilical cord patients, the current and subsequent ultrasound reviews were completed by two fixed obstetric ultrasound experts.

$\nabla$ Measured the blood flow spectrum of the umbilical artery on one or both sides of the fetal bladder. According to the spectrum shape, the umbilical artery was defined as high resistance type and normal type. High resistance type referred to the blood flow spectrum of one or both sides of the umbilical artery, which shows that notch in the early diastolic period and the blood flow velocity increases rapidly in the diastolic period, and none of the above-mentioned was normal(Figure 1)

$\nabla$ To determine whether there was intrauterine growth restriction (FGR), the FGR was used to describe fetuses with an estimated fetal weight less than the 10 th percentile for gestational age ${ }^{[6]}$.

Measured the peak flow velocity of the middle cerebral artery during the diastolic period, and it is defined as faster if bigger than $1.5 \mathrm{MoM}$. The increased peak flow during the diastolic period means the adverse state of fetal hypoxia, which is different from that in systolic period means fetal anemia.

$\nabla$ Amniotic fluid index $(\mathrm{AFI})<5 \mathrm{~cm}$ was used to diagnose oligohydramnios.

$\nabla$ The echo of brain parenchyma was enhanced.

$\nabla$ The spectrum of venous catheter(VC) blood flow was defined as abnormal when there was a reverse A wave.

\section{Pregnancy outcome and follow-up}

According to the delivery situation, the patients were divided into three groups. The patients with severe fetal abnormalities or intrauterine fetal death were terminated pregnancy group; The patients with fetal distress due to abnormal fetal heart rate monitoring and termination of pregnancy by emergency cesarean section were divided into intervention group [7] [8]; The patients with normal fetal heart rate monitoring and no fetal distress were selected as non-intervention group. Reviewed and summarized the prenatal and intrapartum fetal heart rate monitoring, gestational age at delivery, mode of delivery, and neonatal birth weight of the three groups. The birth weight of the newborn was less than the tenth percentile considered to be small for gestational age (SGA). The neonatal growth and development were followed up by telephone.

\section{Statistical methods and the feasibility of establishing HC clinical early warning system}

We used SPSS 22.0 software to analyze the data. The measurement data of normal distribution were expressed by means \pm standard, compared the results by t-test, and the rank-sum test was used for measurement data of non-normal distribution. The counting data were expressed by frequency and was adopted by $\chi 2$ inspection. One-way ANOVA was used to compare the count data among the three groups, and the SNK-Q test was used to compare the mean of measurement data. All the statistical tests were used a two-sided test, $\mathrm{P}<0.05$ considered as a statistically significant difference.

Through the above statistical methods, the pregnancy outcomes of simple and complex HC patients were compared to see whether there were statistical differences. Compared the distribution of 6 ultrasound risk factors in terminated pregnancy group, intervention group, and nonintervention group. According to the statistical significance results in three groups, discussed the feasibility of establishing a clinical early warning system for HC patients. 


\section{Results}

1. General condition of patients

From January 2019 to March 2021, 32786 patients were examined by ultrasound in our hospital, and the incidence of HC was $0.12 \%$ (38/32786). The average age of patients with $\mathrm{HC}$ was $31.9 \pm 2.3$ years. The average gestational week of the first diagnosis was $30.12 \pm 4.86$ weeks. 4 cases were in the terminated pregnancy group, 10 cases in the intervention group, and 15 cases in the non-intervention group (Table 1). There were 9 patients with simple $\mathrm{HC}$ and 20 with complex $\mathrm{HC}$ (Table 2). Except for the patients in the terminated pregnancy group, the patients in the other two groups were reviewed regularly in our hospital every $2 \sim 4$ weeks after the diagnosis of HC. The average examination times were $3.2 \pm 2.4$ times. According to the patients' condition, all newborns were followed up until now (2 25 months), and no obvious abnormality was found in growth and development.

Table 1

comparison of six high-risk factors in three groups in patients with $\mathrm{HC}$

\begin{tabular}{|c|c|c|c|c|c|c|c|c|c|}
\hline Group & $\begin{array}{l}\text { Frequency } \\
N(\%)\end{array}$ & Mean UCI & $\begin{array}{l}\text { High } \\
\text { resistance } \\
\text { type of } \\
\text { the } \\
\text { umbilical } \\
\text { artery } \\
\mathrm{N}(\%)\end{array}$ & $\begin{array}{l}\text { FGR } \\
N(\%)\end{array}$ & $\begin{array}{l}\text { Faster } \\
\text { peak flow } \\
\text { velocity } \\
\text { of the } \\
\text { middle } \\
\text { cerebral } \\
\text { artery in } \\
\text { diastolic } \\
\text { period } \\
\mathrm{N}(\%)\end{array}$ & $\begin{array}{l}\text { Oligohydramnios } \\
\mathrm{N}(\%)\end{array}$ & $\begin{array}{l}\text { Enhanced } \\
\text { echo of the } \\
\text { brain } \\
\text { parenchyma } \\
\mathrm{N}(\%)\end{array}$ & $\begin{array}{l}\text { Reverse } \\
\text { A wave } \\
\text { in VC } \\
\text { N (\%) }\end{array}$ & $\begin{array}{l}\text { Average } \\
\text { number } \\
\text { of high- } \\
\text { risk } \\
\text { factors in } \\
\text { complex } \\
\text { HC }\end{array}$ \\
\hline $\begin{array}{l}\text { Terminated } \\
\text { pregnancy } \\
\text { group }\end{array}$ & $4(13.8 \%)$ & $1.04 \pm 0.19$ & $4(100.0 \%)$ & $4(100.0 \%)$ & $4(100.0 \%)$ & $2(50.0 \%)$ & $2(50.0 \%)$ & $3(75.0 \%)$ & $5.25 \pm 0.99$ \\
\hline $\begin{array}{l}\text { Intervention } \\
\text { group }\end{array}$ & $10(34.5 \%)$ & $0.81 \pm 0.04$ & $5(50.0 \%)$ & $7(70.0 \%)$ & $4(40.0 \%)$ & $3(30.0 \%)$ & $2(20.0 \%)$ & $0(0 \%)$ & $2.38 \pm 1.01$ \\
\hline $\begin{array}{l}\text { Non- } \\
\text { intervention } \\
\text { group }\end{array}$ & $15(51.7 \%)$ & $0.74 \pm 0.06$ & $3(20.0 \%)$ & $4(26.7 \%)$ & $0(0 \%)$ & $0(0 \%)$ & $0(0 \%)$ & $0(0 \%)$ & $1.29 \pm 0.50$ \\
\hline $\mathrm{p}$ & - & 0.000 & 0.012 & 0.011 & 0.000 & 0.026 & 0.028 & 0.000 & 0.000 \\
\hline
\end{tabular}

Table 2

comparison of pregnancy and neonatal status between simple and complex HC patients

\begin{tabular}{|c|c|c|c|c|c|c|c|c|c|}
\hline Group & $\begin{array}{l}\text { Frequency } \\
N(\%)\end{array}$ & Mean UCl & $\begin{array}{l}\text { Gestational } \\
\text { age of the } \\
\text { first } \\
\text { detection of } \\
\text { HC(weeks) }\end{array}$ & $\begin{array}{l}\text { Terminated } \\
\text { pregnancy } \\
\text { N (\%) }\end{array}$ & $\begin{array}{l}\text { Intervention } \\
\mathbf{N}(\%)\end{array}$ & $\begin{array}{l}\text { Abnormal } \\
\text { fetal heart } \\
\text { rate } \\
\text { monitoring } \\
\mathrm{N}(\%)\end{array}$ & $\begin{array}{l}\text { Gestational } \\
\text { age of } \\
\text { delivery(weeks) }\end{array}$ & $\begin{array}{l}\text { Cesarean } \\
\text { section } \\
\mathrm{N}(\%)\end{array}$ & $\begin{array}{l}\text { SGA } \\
N(\%)\end{array}$ \\
\hline $\begin{array}{l}\text { Complex } \\
\text { HC }\end{array}$ & $20(69.0 \%)$ & $0.84 \pm 0.17$ & $30.79 \pm 4.85$ & $4(20 \%)$ & $9(45.0 \%)$ & $9(56.3 \%)$ & $34.55 \pm 4.70$ & $14(87.5 \%)$ & $9(56.3 \%)$ \\
\hline $\begin{array}{l}\text { Simple } \\
\mathrm{HC}\end{array}$ & $9(31.0 \%)$ & $0.72 \pm 0.08$ & $28.64 \pm 4.84$ & $0(0 \%)$ & $1(11.1 \%)$ & $1(11.1 \%)$ & $37.62 \pm 2.62$ & $3(33.3 \%)$ & $0(0 \%)$ \\
\hline$p$ & - & 0.419 & 0.969 & 0.148 & 0.027 & 0.027 & 0.034 & 0.005 & 0.005 \\
\hline
\end{tabular}

3 cases (3/4) in terminated pregnancy group had no amniotic fluid, fetal death, or severe FGR when they were examined in our hospital by ultrasound, and then terminated the pregnancy. The other case (1/4), HC was found with high resistance of bilateral umbilical arteries of bladder and increased diastolic blood flow of middle cerebral artery. After failing to follow the doctor's advice for further examination, she went home. Three days later, she found fetal death in the hospital after the disappearance of fetal movement. Compared with this case in the terminated pregnancy group, two patients in the intervention group also found $\mathrm{HC}$ combined with high resistance of bilateral umbilical arteries of bladder and rapid diastolic blood flow of middle cerebral artery. According to the doctor's advice, they were admitted to the hospital for emergency assessment and found abnormal fetal heart rate monitoring. They had a cesarean section after suspected fetal distress (Figure 2). There was no obvious abnormality in the growth of the newborns. At the same time, the other two patients in the intervention group had transient high resistance of bilateral umbilical arteries of the bladder, increased diastolic blood flow of middle cerebral artery and oligohydramnios, and the above ultrasound abnormalities disappeared after reexamination. All patients in the intervention group (10 cases) were suspected of fetal distress and terminated 
pregnancy early. Among them, 6 cases (6/10) had frequent moderate to severe variable deceleration (VD) before delivery, and 4 cases (4 / 10) were unresponsive to continuous non-stress test( NST). In the non-intervention group, 15 patients had normal fetal heart rate monitoring during pregnancy and perinatal periods.

2. Distribution of ultrasound high-risk factors in terminated pregnancy group, intervention group, and non-intervention group

The distribution proportion of six high-risk factors was statistically different among the three groups. In the terminated pregnancy group, there were 3 cases ( 3 / 4) that had reverse A waves in the VC, and 0 in the other two groups. There was only a high resistance type of umbilical artery and FGR in the non-intervention group. The faster peak flow velocity of the middle cerebral artery in the diastolic period, oligohydramnios, and enhanced echo of brain parenchyma began to appear in the intervention group. And the incidences of these 3 factors in non intervention group, intervention group and terminated pregnancy group were $0 \%, 40 \%$ and $100 \% ; 0 \%, 30.0 \%$ and $50.0 \%$; $0 \%, 20.0 \%$ and $50.0 \%$, respectively. There were also significant differences in the number of high-risk factors between the three groups. The number of high risk factors in terminated pregnancy group > intervention group > non intervention group, $\mathrm{P}=0.000$ (Table 1).

The average $\mathrm{UCl}$ of 29 patients was $0.80 \pm 0.16$, average $\mathrm{UCl}$ of the terminated pregnancy group was $1.04 \pm 0.19$. There was a statistical difference between the terminated pregnancy group to the intervention group and the non-intervention group $(P=0.000)$. Compared with the non-intervention group, the average $\mathrm{UCl}$ of the intervention group was higher, but there was no statistical difference $(P=0.769)$.

3. Comparison of pregnancy outcome and distribution between simple and complex $\mathrm{HC}$ patients

Compared with simple $\mathrm{HC}$ patients, patients with complex $\mathrm{HC}$ had higher proportion of clinical intervention $(P=0.027)$, higher abnormal fetal heart rate monitoring $(P=0.027)$, early gestational age $(P=0.034)$, higher cesarean section rate $(P=0.005)$ and higher proportion of low birth weight $(P=0.005)$. There was no significant difference in the average $\mathrm{UCl}(P=0.419)$, the gestational age of the first detection of $\mathrm{HC}(P=0.969)$ and the proportion of terminated pregnancy $(P=0.148)$ in these two groups.

There were 4 cases (4/4), 9 cases (9/10), and 7 cases (7/15) of complex HC patients in terminated pregnancy group, intervention group, and nonintervention group. And simple $\mathrm{HC}$ patients were only distributed in the intervention group and non-intervention group, 1 case (1 / 9 ) and 8 cases $(8 / 9)$, respectively. The distribution of simple and complex HC patients in the three groups was statistically different $(\mathrm{P}=0.025)$.

\section{Discussion}

Previous ultrasound examination found that $\mathrm{HC}$ may be associated with adverse pregnancy outcomes, and the A wave reverse in VC was the fetal critical value, once it was found that the pregnancy needed to be terminated in time ${ }^{[9]}[10]$. And we found that in $\mathrm{HC}$ patients with A wave reverse in VC, the fetus had serious abnormalities or fetal acute intrauterine distress. Meanwhile, not all patients with HC have adverse pregnancy outcomes

[11]. Therefore, it was necessary to establish an ultrasound early warning system that can provide opportunities for clinical intervention. Ernst et al. classified umbilical cord of $\mathrm{HC}$ in gross patterns. Due to the relatively high incidence of umbilical cord hyper-coiling and to personal, clinical observations that not all hypercoiled cords were associated with evidence of fetal vascular obstruction and/or poor outcome ${ }^{[12]}$. However, Ernst's study focused on the umbilical cord after delivery, when the pregnancy outcome had been determined, it cannot give guidance for clinical intervention during pregnancy. According to the final clinical treatment, 29 patients with HC diagnosed and delivered in our hospital were divided into non-intervention group, intervention group, and terminated pregnancy group, objective to explore the feasibility of establishing an ultrasound early warning system for complex HC patients.

We found that there was no significant difference in the mean UCl between the intervention group and non-intervention group in 29 patients with $\mathrm{HC}$. There was no significant difference in average $\mathrm{UCl}$ between complex $\mathrm{HC}$ and simple $\mathrm{HC}$ patients as well. It indicated that UCl could not be an objective index alone to determine the intervention mode for clinical doctors, which was consistent with the previous literature. But in the terminated pregnancy group, intervention, and non-intervention, there were significant statistical differences in the types and number of high-risk factors, which made them a multivariate factor affecting the clinical treatment and pregnancy outcome of HC patients. The high resistance of umbilical artery and FGR appeared first in the patients, and the incidence of them increased in the non-intervention group, intervention group, and terminated pregnancy group. The faster peak flow velocity of the middle cerebral artery in the diastolic period, oligohydramnios, and enhanced echo of brain parenchyma did not occur in the non-intervention group, and the incidence rate increased in the intervention group and terminated pregnancy group, while the A wave reverses in VC only occurred in the terminated pregnancy group, It indicated that it was related to the terminal stage of the fetus. The average number of high-risk factors was also statistically significant among the groups. Therefore, we thought that high resistance of umbilical artery and FGR may be the earliest warning indicators for HC patients who need clinical intervention. For HC fetuses with this manifestation, we should further closely observe the peak flow velocity of the middle cerebral artery in the diastolic period, amniotic fluid volume, and the echo of brain parenchyma, to grasp the timing of clinical intervention, and avoided further deterioration of the fetal condition and lost the intervention time. When $\mathrm{UCl}>1$ or the number of high-risk factors was more than 5 or the VC A wave was reversed, the children usually had adverse pregnancy outcomes, and clinical intervention was needed. 
Once the patient was in the clinical early warning system, the gestational age of delivery was earlier, because the average gestational age of delivery in patients with complex $\mathrm{HC}$ was $34.55 \pm 4.70$ weeks, and the possibility of cesarean section increased. Therefore, for patients with early warning indications, we needed to contact clinical obstetricians for attention. We could take fetal lungs' maturity-accelerating therapy in advance, strengthen the assessment of fetal safety and the frequency of fetal heart rate monitoring, make preparations for timely detection of fetal distress and early delivery, and avoid missing the opportunity of treatment ${ }^{[7]}$. Through the comparison of different obstetric treatment and pregnancy outcomes between a patient in the terminated pregnancy group and two patients in the intervention group, we found that once ultrasound found abnormal indicators, timely clinical intervention could improve the pregnancy prognosis.

In several times of ultrasound examinations, we found that the above-mentioned ultrasound signs of $\mathrm{HC}$ patients may change, which may gradually change from intermittent to continuous, or may return to normal intermittently. Therefore, the ultrasound indicators cannot be used as the indicators to terminate the pregnancy, but still needed to be considered in combination with clinical conditions such as fetal movement and fetal heart rate monitoring. Once a certain index continued to appear abnormal, we need to be vigilant, which indicated that the $\mathrm{HC}$ of the umbilical cord increases the possibility of poor prognosis of the fetus. In the intervention group, frequent moderate to severe VD often occurred in fetal heart rate monitoring, and the occurrence of VD in fetal heart rate monitoring was related to umbilical cord compression. So we considered that the HC of the umbilical cord led to the increase of umbilical cord pressure and the decrease of umbilical cord blood flow, and its mechanism was similar to the physical pressure of umbilical cord prolapsed, so it would lead to the occurrence of VD.

Because the incidence rate of $\mathrm{HC}$ is low, the number of cases was limited, further studies were needed.

\section{Conclusions}

In conclusion, the following high-risk factors should be further evaluated when $\mathrm{HC}$ was found in patients: $\nabla$ High resistance of umbilical artery on one or both sides of bladder, $\triangle F G R, \otimes$ Faster peak flow velocity of middle cerebral artery increased in diastolic phase, $\varangle A F I \nabla 5 \mathrm{~cm}, \nabla$ Enhanced echo of brain parenchyma, $\triangle$ Reverse A wave in $\mathrm{VC}$, and these six items were defined as the evaluation high-risk factors of $\mathrm{HC}$ clinical early warning system. Once one of them was combined, it was considered as complex HC. In patients with complex HC, the proportion of intervention was increasing, the gestational age of delivery was earlier, the fetal heart rate monitoring was often needed as early as possible, the pregnancy should be terminated as soon as possible when the fetal heart rate monitoring was abnormal, the cesarean section rate was higher, and the incidence of SGA was higher, which all needed to cause a clinical warning. The more complicated HC patients with high-risk factors, the worse pregnancy prognosis may be. When the UCl was greater than 1 and the number of high-risk factors was greater than 5 , the probability of adverse pregnancy outcomes was high. High resistance of unilateral or bilateral umbilical artery and FGR were the earliest warning indications in the early warning system. Once it was found that clinical intervention was needed in time, effective obstetric intervention and management could improve the prognosis of pregnancy.

\section{Declarations}

\section{Statement of guidelines and regulations.}

We confirmed that all methods were carried out in accordance with relevant guidelines and regulations. Ultrasound screening was performed according to the routine and regulations of prenatal ultrasound screening in the Peking University People's Hospital, and the following references

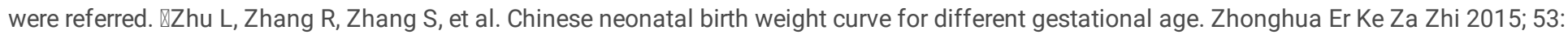
97-103 (in Chinese). खFetal Medicine Subgroup, Society of Perinatal Medicine, Chinese Medical Association, Obstetrics Subgroup, Society of Obstetrics and Gynecology, Chinese Medical Association. Expert consensus on fetal growth restriction. Chin J Perinat Med,2019;22(6):361-380. ( in Chinese).

\section{Ethics approval and consent to participate}

The study was approved by the Institutional Review Boards of Peking University People's Hospital (2016PHB054-01).

Informed consent was obtained from all pregnant women (all adults) examined in our hospital.

\section{Consent for publication}

Not Applicable

\section{Availability of data and materials}

The datasets generated and analysed during the current study are not publicly available due to limitations of ethical approval involving the patient data and anonymity but are available from the corresponding author on reasonable request.

\section{Competing interests}


The authors declare that they have no known competing financial interests or personal relationships that could have appeared to influence the work reported in this paper.

\section{Funding}

Our study had no funding source.

\section{Authors' contributions}

Junshu Xie, Xiaohong Zhang and Qiuyan Pei wrote the main manuscript text and Yuntao Li, Yani Yan,Zhenjuan Yang and Xiaowei Xue collected data and prepared figures 1-2. All authors reviewed the manuscript.

\section{Acknowledgements}

Thank all the staff of the Department of Obstetrics and Gynecology of Peking University People's Hospital for their support for our research work.

\section{References}

[1] Strong Jr TH, Jarles DL, Vega JS, et al. The umbilical coiling index. Am J Obstet Gynecol Jan 1994;170(1):29-32.

[2] Wilke DJ, Denier JP, Khong TY, et al. Estimating umbilical cord flow resistance from measurements of the whole cord. Placenta. 2021;103:180187.

[3] F.A. Jessop, C.C. Lees, S. Pathak, et al, Umbilical cord coiling: clinical outcomes in an unselected population and systematic review, Virchows Arch. 2014;464 (1) :105-112.

[4] N.J. Sebire, Pathophysiological significance of abnormal umbilical cord coiling index, Ultrasound Obstet. Gynecol. 2007;30 (6): $804-806$.

[5] Salomon LJ, Alfirevic Z, Berghella V, et al. Practice guidelines for performance of the routine mid-trimester fetal ultrasound scan. Ultrasound Obstet Gynecol 2011; 37: 116-126.

[6] Zhu L, Zhang R, Zhang S, et al. Chinese neonatal birth weight curve for different gestational age. Zhonghua Er Ke Za Zhi 2015; 53: 97-103 (in Chinese)

[7] National Collaborating Center for Women's and Children's Health, Intrapartum care: Care of healthy women and their babies during childbirth. National Collaborating Centre for Women's and Children's Health,2007;217-228

[8] American College of Obstetricians and Gynecologists. ACOG Practice Bulletin No.106: Intrapartum fetal heart rate monitoring: Nomenclature, interpretation, and general management principles. Obstet Gynecol, 2009;114(1):192-202

[9] Bragaa M, Moleirob ML, Guedes-Martins L. Clinical Significance of Ductus Venosus Waveform as Generated by Pressure-volume Changes in the Fetal Heart, Current Cardiology Reviews, 2019;15:167-176

[10] Fetal Medicine Subgroup, Society of Perinatal Medicine, Chinese Medical Association, Obstetrics Subgroup, Society of Obstetrics and Gynecology, Chinese Medical Association. Expert consensus on fetal growth restriction. Chin J Perinat Med,2019;22(6):361-380. (in Chinese)

[11] T.Y. Khong. Evidence-based pathology: umbilical cord coiling. Pathology. 2010;42(7):618-622

[12] Ernst LM, Minturn L, Huang MH, et al. Gross patterns of umbilical cord coiling: Correlations with placental histology and stillbirth. Placenta,2013;34:583-588

\section{Figures}



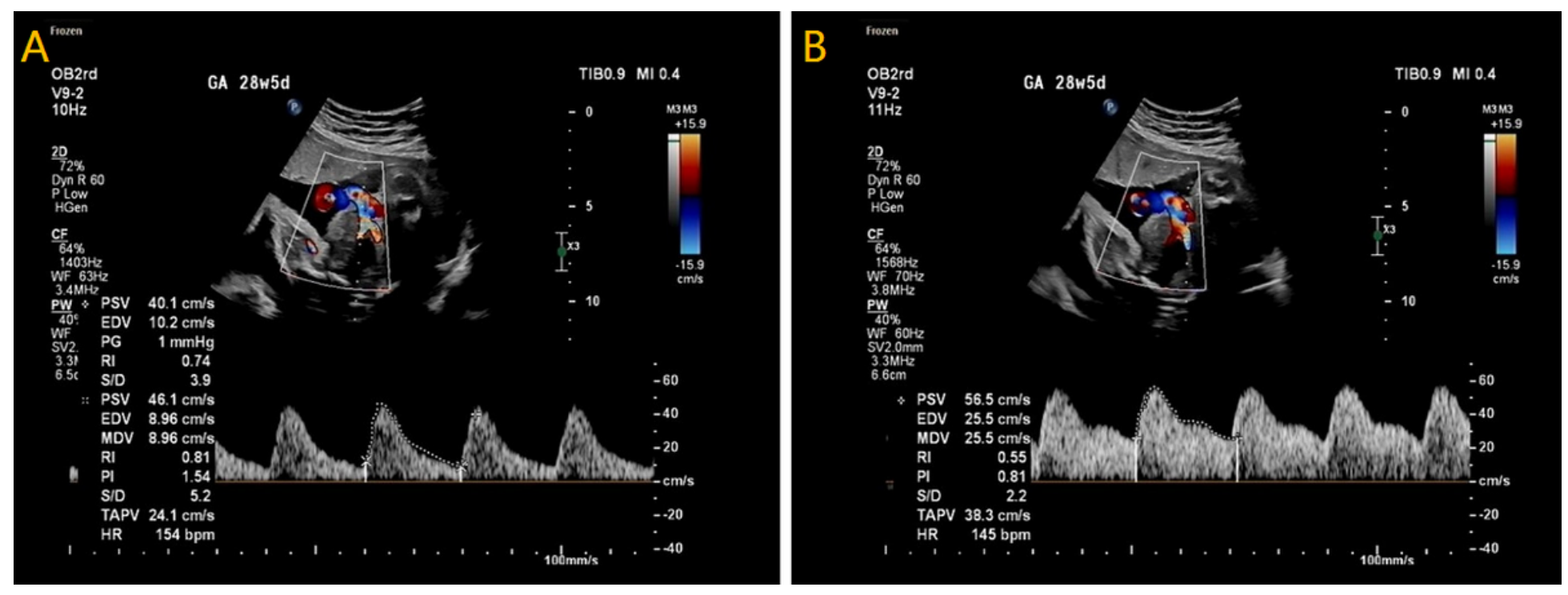

\section{Figure 1}

The spectrum of umbilical artery on both sides of the bladder in the same patient (Figure A: Normal spectrum of umbilical artery. Figure B: The notch of umbilical artery spectrum in early diastolic period and the increased flow velocity of the diastolic period, i.e. high resistance type of umbilical artery.)
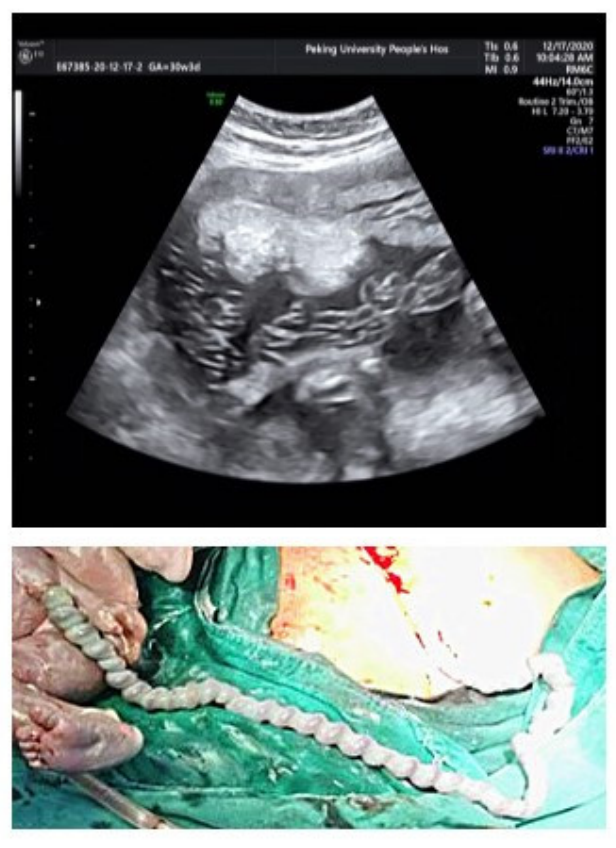

Figure 2

$\mathrm{HC}$ image of ultrasound and the shape of the umbilical cord after birth in the intervention group 